\title{
A Case Series of Congenital Ovarian Cyst in A Tertiary Care Hospital in Mumbai, India
}

\author{
Bhavana Madhukar Bharambe*, Saroj Ashok Bolde, Sushma Chandeshwar Bharti and Neha Satyanarayan Somani \\ Department of Pathology, Grant Government Medical College, Mumbai, India
}

\section{ABSTRACT}

The congenital ovarian cysts are one of the most frequent lesions to be diagnosed antenatally. The manifestations postnatally determine the line of management of the cysts. These can manifest as abdominal lump, torsion or may be asymptomatic.

We present a case series of four cases of congenital ovarian cyst presenting to our tertiary care hospital.

Keywords: Ovarian Cyst, Congenital Cyst, Antenatal Ovarian Cyst, Childhood Ovarian Cyst

\section{Introduction}

Congenital ovarian cyst is a rare clinical finding seen in infants. This entity was first described by Valenti et $\mathrm{al}^{[1]}$ in 1975 . These cysts are present prenatally and are increasingly being diagnosed in fetal or postnatal period by various diagnostic modalities of the modern era, especially antenatal ultrasonography (USG) and magnetic resonance imaging (MRI). The ovarian cysts can be symptomatic or asymptomatic. They are classified into simple and complex radiologically by Nussbaum et al. ${ }^{[2]}$ These are usually unilocular and unilateral.

We present four cases of congenital ovarian cysts with varied manifestations and histomorphology.

\section{Case Report(S)}

Case 1: A 14 day female child born to 26 year old mother was diagnosed with left ovarian cyst in the antenatal scan. The cyst was sized $2.8 \times 2.2 \times 1.5 \mathrm{~cm}$ and was unilocular. The remaining investigations were normal.

Case 2: A 27 day old child was bought to pediatric surgery department with complaints of crying spells and vomiting. The patient had left lumbar and iliac tenderness and guarding of abdomen. On USG, a twisted complex ovarian cyst sized $5.5 \times 3.7 \times 4.2 \mathrm{~cm}$ was seen on left side with tiny cystic spaces within. The child was immediately operated upon for derotation of ovarian cyst with deroofing done. Bilateral oophoropexy was done. Postoperatively, patient is doing well and is healthy.

Case 3: A one month and 6 day old female child was bought by her 25 year old mother with complaints of constant crying. On examination, tenderness was noted in abdomen on left side. USG abdomen showed well defined cystic lesion in left lumbar region suggestive of mesenteric or ovarian cyst. Contrast enhancing computed tomography (CECT) abdomen showed a well defined cystic lesion suggestive of ovarian teratoma or enteric duplication cyst. Diagnostic laparoscopy revealed twisted ovarian cyst which was removed and sent for histopathology. The child was stable after surgery.

Case 4: A full term, one day old female child, born to a primigravida was admitted with complaints of a pelvic cyst. The cyst was diagnosed prenatally by USG. It was a well defined cyst sized $4 \times 4.8 \times 4.9 \mathrm{~cm}$ on left side showing septations. The ovarian cystectomy was performed with oophorectomy.

None of the above babies had any associated congenital malformation as described in literature.

The gross and histopathological examination of the received ovarian cysts confirmed the ovarian origin with focally preserved flattened to cuboidal lining epithelium. There was evidence of dystrophic calcification in all the cases along with stromal hemorrhages (Fig. $1-3$ ).

\section{Discussion}

The term congenital ovarian cyst encompasses all the ovarian cysts detected around neonatal period. Though exact age limit hasn't been proposed in the literature. The retrospective evaluation of still borns and newborns with death within 4 weeks of birth suggests that the overall incidence of congenital ovarian cyst is approximately $30 \% \cdot{ }^{[3]}$ The other intra-abdominal cysts which are common include ureterocele, urachal cyst, hydrocolpos, renal cysts, enteric duplication cyst, meconium cyst, lymphangioma and fetus in fetu.

These ovarian cysts are believed to arise from the persistent effect of maternal gonadotrophin levels on the fetal ovarian 


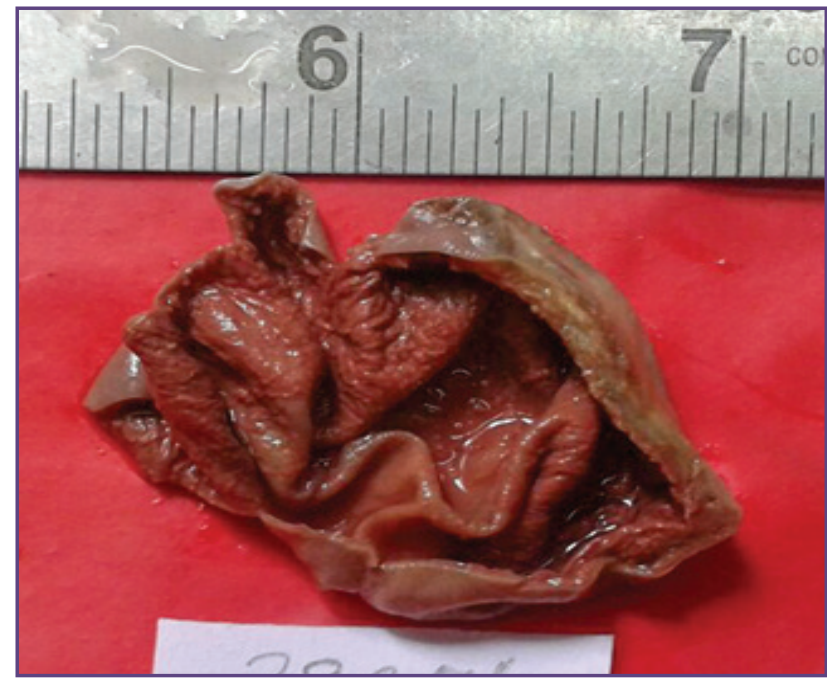

Fig. 1: A unilocular cyst with smooth external surface and hemorrhagic inner lining.

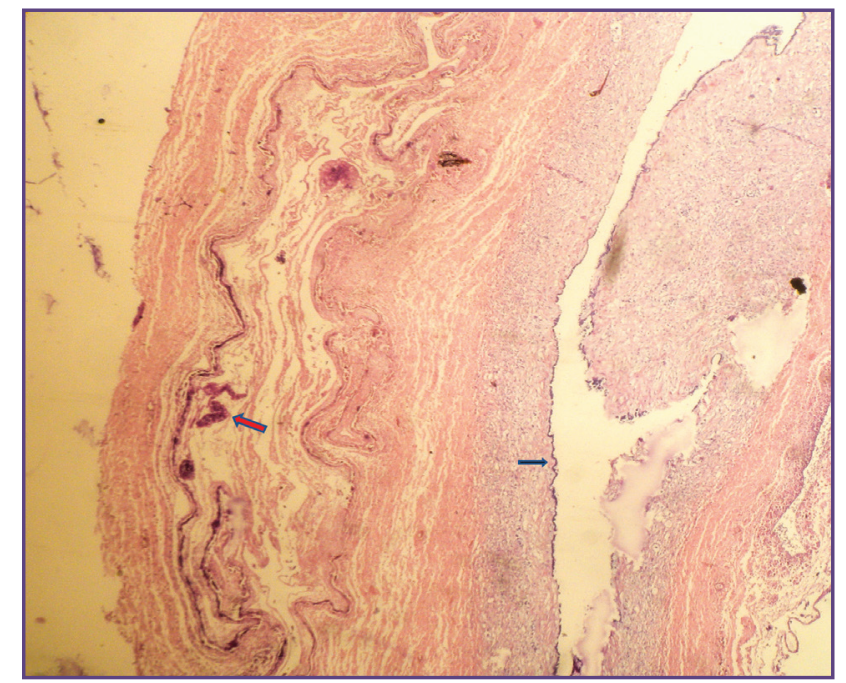

Fig. 2: Shows dystrophic calcification (red arrow) and focally ulcerated flattened epithelial lining (blue arrow). (H\&E, X 400).

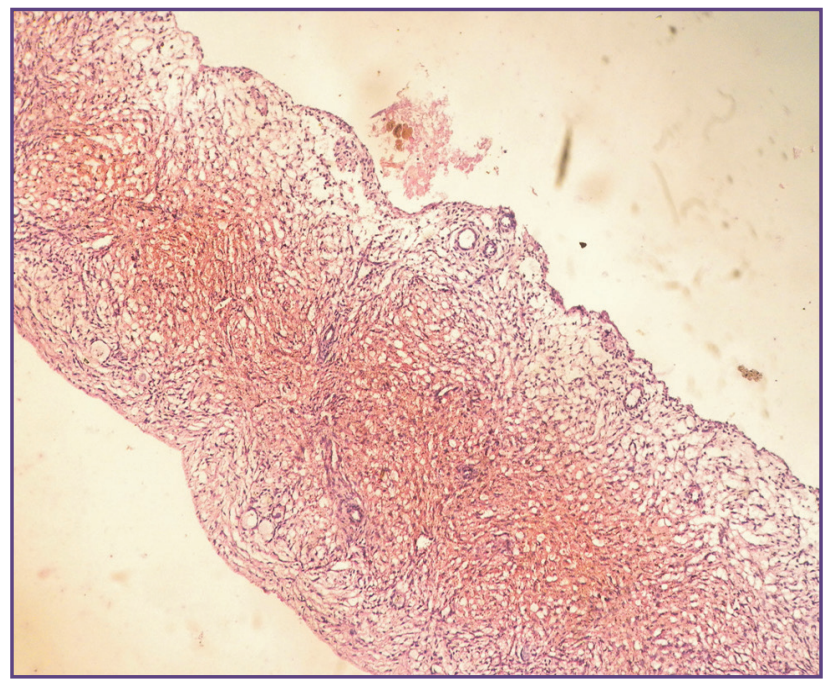

Fig. 3: Shows haemorrhages in the cyst wall. (H\&E, X 400).

tissue resulting in the stimulation of a naive ovarian follicle causing a cystic transformation. ${ }^{[4]}$ These cysts are earliest detected on prenatal imaging done as routine ultrasonography evaluation and can be picked up as early as $28^{\text {th }}$ week of gestation. However, all cysts identified on prenatal ultrasound do not need an intervention. The need to actively intervene depends on serial documentation of features which act as predictive markers for development of complications significant to affect the life of neonate. The most common complication to arise in congenital ovarian cyst is development of torsion of the ovarian pedicle which forms 50 to $70 \%$ of all complications. ${ }^{[4,5]}$ The remaining complications being haemorrhage, rupture, peritionitis secondary to inflammation, urinary tract obstruction, bowel obstruction, autoamputation and in rare case of sterile necrosis of the ovary. These congenital ovarian cysts are known to undergo spontaneous regression.

Several studies have tried to evaluate the predictive markers in prenatal ultrasonography which can predict an increased risk of developing complication and planning an active intervention. Such predictive markers on prenatal USG include length of the ovarian pedicle, size of the cyst, laxity of the ovarian and infundibular ligament, solitary or multiple cyst in the same ovary, unilateral or bilateral involvement, simple versus complex nature of cyst, presence /absence of increased vascularity or congestion of ovarian pedicle on colour doppler. ${ }^{[5]}$ Torsion of ovarian pedicle does result in inflammatory process reflected 
by subtle rise in alpha foeto protein/carcinoembryonic antigen levels but they have not been found to be predictive in identifying development of complications in congenital ovarian cysts. The most important markers on prenatal ultrasonogram to predict a complication include size and eco pattern of the cyst. A simple uniform solitary cyst of size less than $4 \mathrm{~cm}$ is unlikely to give rise to any complication, however such cyst have a potential for undergoing a reduction with progression of pregnancy and spontaneous resolution. This advocates a serial ultrasonographic evaluation in prenatal phase, at weekly interval to document the progress of cyst with pregnancy. Bagolan et al. managed 34 simple ovarian cysts $<5 \mathrm{~cm}$ with wait and see policy and have observed spontaneous regression in 26 cases, 1 persistent at birth and 7 cases of torsion. ${ }^{[6,7]}$ Certain studies report conservative management for simple cyst upto size of $8 \mathrm{~cm}$. Intrauterine ovarian cyst aspiration remains another promising option for management of congenital ovarian cyst. However, the risk associated with invasive procedure, the potential to cause haemorrhage, rupture/ spillage with inflammation, or infection does merit a careful selection of patents to be considered for aspiration. Bagolan et al in their study performed in utero aspiration of fourteen simple cysts measuring $5 \mathrm{~cm}$ observing resolution in twelve cases and torsion in two concluding that "in utero" aspiration of ovarian cyst is a safe procedure. ${ }^{[6,7]}$ Cases of congenital ovarian cysts which present with torsion or rupture warrant a surgical exploration either open or minimal invasive approach. In cases where complex ovarian cyst appears likely, ultrasonography may not be reliable and CT scan or MRI is more informative to define the nature of cyst. The time to consider for surgical intervention is also influenced by the extent of fetal lung maturation to salvage the cystic ovary. Most of the cases present as unilocular cysts which histologically may be follicular cyst, simple cyst, theca lutein cyst or corpus luteum cyst with characteristic presence of dystrophic calcification and haemorrhages in the cyst wall. The lining epithelium may or may not be seen.
In our study, all cases were symptomatic and all presented in post natal period under the age of $2 \mathrm{yrs}$ with symptoms of abdominal pain. All the patients underwent surgical procedure in view of ultrasonography findings, symptomatic presentation and development of complications. The retrospective evaluation of clinical spectrum and histopathology reveals presence of cyst in prenatal ultrasonography which subsequently underwent complication requiring surgical intervention.

\section{Conclusion}

Congenital ovarian cyst is a significantly prevalent finding on prenatal ultrasonography, which usually requires a regular sonographic monitoring. Intervention is only required in patients with complications or those who are at potential risk for developing complications as evaluated on serial monitoring. Overall prognosis for all congenital ovarian cysts remains excellent.

\section{Reference}

1. Valenti C, Kassner EG, Yermakow V, Comb E. Antenatal diagnosis of a fetal ovarian cyst. Am J Obstet Gynecol 1975;15:216-219.

2. Nussbaum AR, Sanders RC, Hartmann DS. Neonatal ovarian cysts: sonographic-pathologic correlation. Radiology 1988; 168:817-821.

3. Anna Dera-szymanowska, Mariolaropacka-lesiak, Michałbłaszczyński, Marta Szymankiewicz, Grzegorz H. Bręborowicz. Torsion of adnexal cyst in utero - case report. Archives of Perinatal Medicine 2012;18,229-232.

4. Mudholkar V, Acharya A, Kulkarni A, Hirgude S. Antenatally diagnosed neonatal ovarian cyst with torsion. Indian Journal of Pathology and Microbiology 2011;54:228- 229.

5. Ibrahim H, Lewis D, Harrison GK, Tice H, Sangster G. Journal of Perinatology 2007;27,523-526.

6. Bagolan P, Rivosecchi M, Giorlandino C, Bilancioni E, Nahom A, Zaccara A, et al. Prenatal diagnosis and clinical outcome of ovarian cysts . J Pediatr Surg 1992;27:879-881.

7. Bagolan P, Giorlandino C, Nahom A, Bilancioni E, Trucchi A, Gatti C, et al. The management of fetal ovarian cysts. J Pediatr Surg 2002;37:25-30.

*Corresponding author:

Dr. Bhavana Madhukar Bharambe, Flat 32, Trimurti Bldg, JJ Hospital Campus, Byculla, Mumbai-400008, India

Phone: +91 9892906747

Email: bhavanab.136@gmail.com

Financial or other Competing Interests: None.

Date of Submission : 07.11.2016

Date of Acceptance : 03.02.2017

Date of Publication : 28.03.2017 